\title{
How to be a mitotic chromosome
}

\author{
Sandra C. Moser • Jason R. Swedlow
}

Published online: 2 April 2011

(C) The Author(s) 2011. This article is published with open access at Springerlink.com

\begin{abstract}
Proper mitotic chromosome structure is essential for faithful chromosome segregation. Mounting evidence suggests that mitotic chromosome assembly is a progressive, dynamic process that requires topoisomerase II, condensins and cohesin and the activity of several signalling molecules. Current results suggest how these different activities might interact to achieve the familiar form of the mitotic chromosome.
\end{abstract}

Keywords chromosome structure $\cdot$ condensation . condensin $\cdot$ topoisomerase II $\cdot$ cohesin

$\begin{array}{ll}\text { Abbreviations } \\ \text { CAP } & \text { Chromosome-associated protein } \\ \text { CDC } & \text { Cell division cycle } \\ \text { CDK } & \text { Cyclin-dependent kinase } \\ \text { FRAP } & \text { Fluorescence recovery after photobleaching } \\ \text { GFP } & \text { Green fluorescent protein } \\ \text { HP1 } & \text { Heterochromatin protein } 1 \\ \text { Kif4a } & \text { Kinesin family member 4a } \\ \text { Pds } & \text { Precocious dissociation of sisters } \\ \text { Plk1 } & \text { Polo-like kinase 1 }\end{array}$

Responsible Editors: James Wakefield and Herbert Macgregor.

S. C. Moser $(\bowtie) \cdot J$. R. Swedlow

Wellcome Trust Centre for Gene Regulation and Expression, College of Life Sciences,

University of Dundee,

Dundee DD1 5EH, UK

e-mail: s.c.moser@dundee.ac.uk
PP2A Protein phosphatase 2A

RCA Regulator of chromosome architecture

Rec8 Recombination 8

RNAi RNA interference

SA Stromal antigen

SILAC Stable isotope labeling with amino acids in cell culture

Sc1 Scaffold protein 1

SCC Sister chromatid cohesion

Sgo1 Shugoshin 1

SMC Structural maintenance of chromosomes

SUMO Small ubiquitin-like modifier

Topo Topoisomerase

Wapl Wings apart-like

\section{Introduction}

The genomic information of all eukaryotes is stored on long strands of DNA called chromosomes. These strands are orders of magnitude longer than individual cells and must be assembled into higher-order structures that substantially reduce chromosome length and organize the chromosome into functional domains appropriate for the fundamental processes of transcription, replication and segregation. The organization of chromosomal DNA is particularly important in mitosis. The correct segregation of chromosomes to daughter cells depends on the proper assembly of kinetochores onto centromeres to mediate the 
connections with the mitotic spindle. Defects in chromosome assembly inevitably lead to a 'cut' phenotype, where chromosome arms are torn by the cleaving cell (Uemura and Yanagida 1984; Saka et al. 1994; Strunnikov et al. 1995). The proteins involved in assembling chromosomes and their functions are highly conserved throughout all eukaryotes.

In this review, we revisit the main non-histone proteins associated with mitotic chromosomes and their proposed roles in mitotic chromosome structure. We also discuss how histone modifications might influence chromosome condensation and how the mitotic chromatin influences the chromosome movement on the mitotic spindle.

Chromosome architecture- of loops, folds and scaffolds

One of the earliest models of chromosome architecture was the radial loop model, in which loops of the 30-nm chromatin fibre were anchored to an axial proteinaceous core (Stubblefield and Wray 1971; Paulson and Laemmli 1977). This model was supported by electron microscopy studies of isolated mitotic chromosomes, clearly showing chromatin loops emanating from a central dense core (Paulson and Laemmli 1977; Marsden and Laemmli 1979). This core could be isolated by digesting mitotic chromosomes with nucleases. Remarkably, the shape and size of the extracted chromosome core matched that of the native, unextracted chromosome. This observation led directly to the concept that this residual structure might reflect the remnants of a non-histone protein 'scaffold' (Paulson and Laemmli 1977; Laemmli et al. 1978; Earnshaw and Laemmli 1983). This term might have been unfortunate - the original description of the scaffold envisioned a loose, interconnected, possibly even dynamic network (Earnshaw and Laemmli 1983); yet, the nomenclature suggested a fixed, structural feature.

The dynamic view of the chromosome was correct30 years of research have revealed the chromosome to comprise a number of ATPases and various enzymes mediating changes in phosphorylation, ubiquitination and SUMOylation, all of which are in constant exchange with a cytoplasmic pool. The only fixed components of chromosomes seem to be the histones themselves, and these are probably dynamic as well — changes in acetylation, phosphorylation and methylation are likely the rule and not the exception, making all features of chromosome architecture chemically and/or structurally dynamic. In many cases, these changes occur in an ordered and interdependent fashion, making it difficult to dissect the function of any individual chromosome component.

So how is a mitotic chromosome built? A progressive winding and folding of chromatin fibres was first postulated based on electron micrographs of moderately thick sectioned material (Sedat and Manuelidis 1978). Improved methods of directly visualizing chromatin in intact cells have consistently supported this view. Belmont's group pioneered the use of tandem arrays of bacterial repressor sequences in cells and expression of repressors fused to a fluorescent protein marker (Belmont et al. 1987, 1999; Strukov and Belmont 2009). These marks can be used as reporters of the location and structure of specific chromosomal regions and are now one of the mainstays of chromatin cell biology and provide the opportunity to visualise a defined sequence in fixed and living cells. Careful examination of these reporters has consistently revealed looped and folded structures that progressively fold to form higher-order structures (Strukov and Belmont 2009).

This concept agrees well with one of the most consistent observations of chromosome architecturethere is no single, constant chromosome structure. Chromosome formation is visible in $\mathrm{G} 2$ cells as the width of condensing fibres grows. In a normal animal cell mitosis, visible shortening and thickening of chromosome arms continues through metaphase and only reverses after chromosome segregation and the initiation of nuclear envelope reassembly. However, extending mitosis through any mechanism that engages the spindle checkpoint - e.g., with spindle poisonscauses continuous thickening and shortening of chromosome arms, until the characteristic, X-shaped structures visible in preparations of karyotypes are visible (Rieder and Palazzo 1992) (Fig. 1a). Thus, chromosome compaction is a continuous process and at the initiation of anaphase, chromosomes have achieved some level of compaction that shortens them sufficiently to clear the cytokinetic furrow and escape the generation of a 'cut' phenotype (Hirano et al. 1986). The key goal of the field is, therefore, the definition and understanding of the molecular and chemical determinants of this dynamic, continuous process. 
Fig. 1 Model for chromosome condensation. a Upon prolonged arrest with spindle poisons mitotic chromosomes become hypercondensed.

DAPI staining of

chromosome spreads of unarrested (left) and nocodazole arrested (right) cells. b Model for chromosome condensation mediated by topo II, c condensins and $\mathbf{d}$ histone modifications. Scale bar $5 \mu \mathrm{m}$
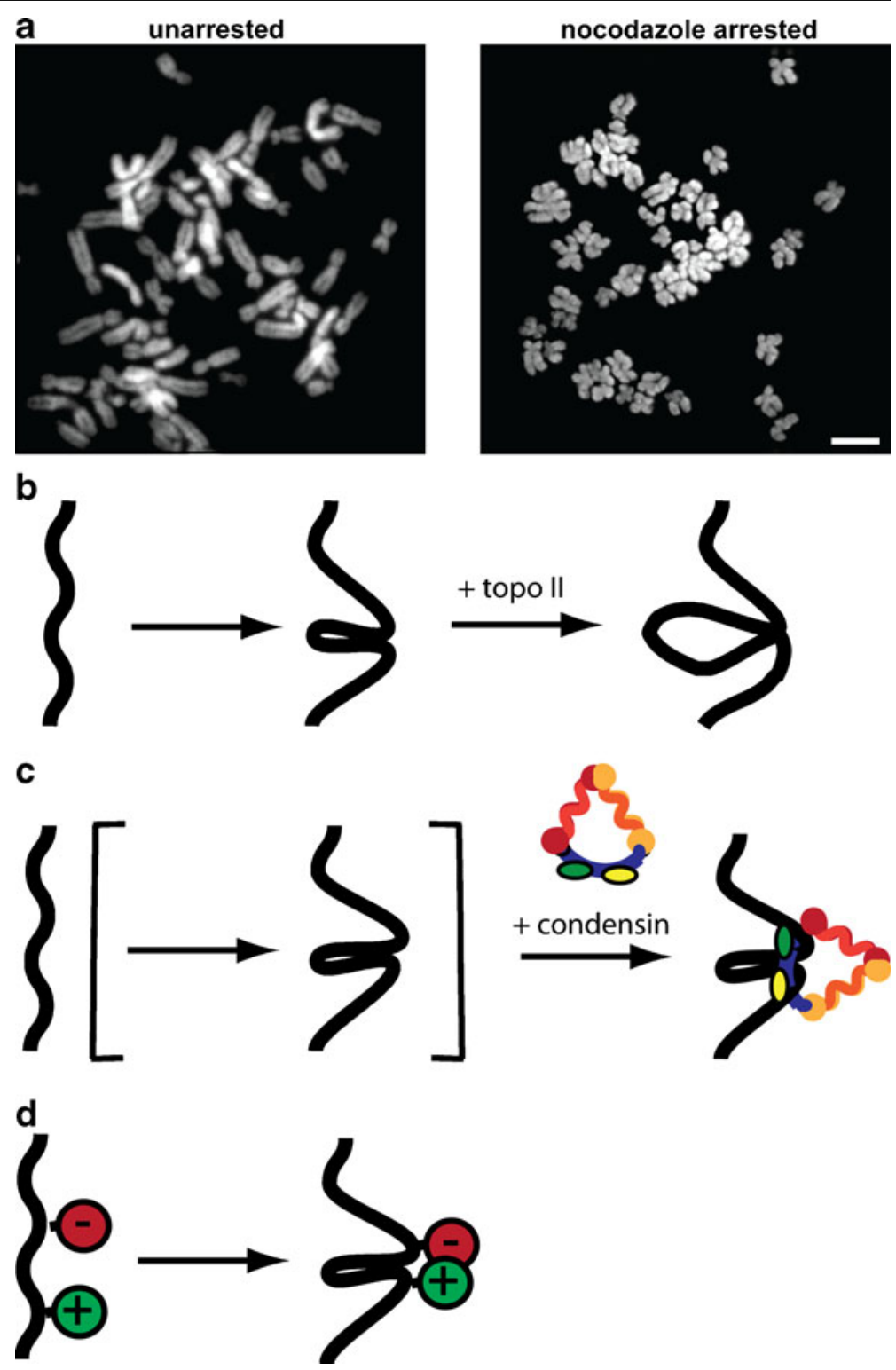

Chromosome biophysics

A number of recent studies have focused on probing the properties of single chromosomes, isolated and manipulated in vitro. Single isolated mitotic chromosomes are elastic and can be reversibly stretched up to five times their original length without obvious perturbation of their structure (Poirier et al. 2000). Spraying single chromosomes with non-specific nucleases (such as micrococcal nuclease) causes their structure to lose all elasticity, highlighting the importance of the DNA in chromosome architecture. Exposure to a 4-bp cutter produces similar effects as digestion with micrococcal nuclease, but a 6-bp cutter has very little effect (Poirier and Marko 2002). Exposure to proteinase $\mathrm{K}$ causes an expansion of chromosomes but never cuts the chromosome and has only a small effect on elasticity. Expansion of chromosomes treated with proteases is anisotropic, with a greater increase in length than width. These simple experiments highlight the key role of DNA in defining the overall structure and the elasticity of 
the chromosome. Chromosome proteins serve to mediate the overall compaction and shortening of the chromosome.

Mitotic chromosomes are highly charged assemblies of macromolecules, containing the negatively charged phosphate backbone of DNA, positively charged histone molecules and many polar non-histone proteins. It, therefore, seems likely that the properties of chromosomes will be affected by interactions with ions in the cytoplasm. Divalent cations or polyamines, at concentrations present in living cells, can determine the folding of chromatin fibres in isolated nuclei (Belmont et al. 1989). Removal or chelation of these ions causes a substantial unfolding of chromatin, confirming a critical role for electrostatics in defining the structure of chromosomes in situ. Similar effects were observed using in vitro chromosome assembly systems based on cell-free Xenopus egg extracts (Hirano and Mitchison 1991). Together, these biophysical and biochemical studies suggest that the overall folding of the chromosome is determined by the DNA and its interaction with the solvent and that chromosome proteins serve to aid compaction and especially to drive the shortening of the chromosome.

\section{Chromosome biochemistry}

Initial biochemical studies identified the major nonhistone protein components of mitotic chromosomes as topoisomerase II, the SMC (structural maintenance of chromosomes) proteins (Earnshaw et al. 1985; Earnshaw and Heck 1985; Gasser et al. 1986; Saitoh et al. 1994). Subsequent work has defined the specific roles of each of these proteins in chromosome structure and function (see below). With the availability of modern proteomic methods, it has been possible to assemble a much more complete list of chromosome proteins from a variety of sources. In any analysis of chromosomes, it is critical to minimise or at least account for any contaminants from the cytoplasm, membranes and other cellular compartments. The Earnshaw lab has addressed this problem using SILAC and mass spectrometry (Ohta et al. 2010). This proteomic analysis yielded many thousands of proteins as candidate chromosome components. Proteins were only scored as truly chromosomal when the amount on chromatin was significantly higher than in the cytosolic fraction and where the domains contained in each protein did not predict an obvious association with another compartment, e.g. plasma membrane. A number of novel candidates were characterised and demonstrated to be bona fide chromosomal proteins by expression of GFP (green fluorescent protein) fusions in cells. This very powerful approach has identified over 2,000 proteins as components of mitotic chromosomes and provides an important resource for future work.

Another system which has proven to be quite powerful in addressing mitotic chromatin structure is the Xenopus cell-free system which enables a reconstitution of mitotic chromosomes from sperm chromatin under physiological conditions in vitro (Hirano and Mitchison 1993). This system has been classically used for functional analysis combining in vitro reconstitution of chromosomes with immunodepletion or antibody addition. It provided the first biochemical characterization of SMC proteins and demonstrated their requirement for proper chromosome assembly (Hirano and Mitchison 1994) and was used to identify a chromosome-associated histone $\mathrm{H} 3$ kinase activity as Aurora B (Murnion et al. 2001). However, more comprehensive analyses are also possible. For example, comparison of interphase and mitotic chromatin proteomes combined with a secondary screen based on live cell imaging has been used to identify novel mitotic kinetochore proteins required for proper chromosome biorientation (Porter et al. 2007).

The ability to recapitulate cell cycle transitions in the Xenopus cell-free system in vitro can also be used for chromatin analysis. Combined with mass spectrometry, it permits the identification of proteins that associate with chromatin in a cell-cycle-dependent manner. To date, this approach has been used to monitor the dynamics of the chromatin proteome during DNA replication (Khoudoli et al. 2008) but can be useful to identify factors that associate with chromatin during the progression from G2 to M and allows the study of the macromolecular dynamics that accompany chromosome formation.

Topoisomerase 2 the disentangler?

A major component of the scaffold known as Sc1 was later identified as topoisomerase II (Lewis and Laemmli 1982; Earnshaw et al. 1985; Gasser et al. 1986). Type II topoisomerases (topo II) are large ATP-dependent homodimeric enzymes that introduce a transient double-strand break into DNA and then pass a second 
DNA strand through the break before resealing it. Numerous studies have shown that topo II is required for normal mitotic chromatin condensation (Uemura et al. 1987; Wood and Earnshaw 1990; Adachi et al. 1991; Hirano and Mitchison 1991). Although topo II was originally identified as part of the chromosome scaffold and, therefore, assumed to be a structural component of the mitotic chromosome, several observations suggest otherwise. Chemical inhibition of topo II which blocks the enzymatic activity but does not affect its binding to chromatin still disturbs chromosome condensation (Rattner et al. 1996). In addition, topo II's association with chromatin is dynamic. The association of topo II with chromosomes varies throughout the cell cycle, reaching its peak in prometaphase (Swedlow et al. 1993). The dissociation of topo II from chromosomes appears to occur in two waves. The first pool of topo II dissociates from chromatin after chromosome segregation, whereas the second pool leaves chromosomes after the completion of mitosis. Two separate isoforms are present in human cells; during mitosis, the alpha isoform (topo IIa) is found on chromosomes, while only a small proportion of the beta isoform is associated with mitotic chromatin (Meyer et al. 1997; Christensen et al. 2002; Null et al. 2002). Fluorescence recovery after photobleaching (FRAP) experiments revealed that topo IIa is rapidly exchanged with the cytoplasmic pool (Tavormina et al. 2002) indicating that topo II is unlikely to be a structural component.

So how does topo II contribute to mitotic chromosome condensation? Biophysical experiments on isolated newt chromosomes suggested that the shape of the mitotic chromosome is to a large extent due to DNA interconnections (Poirier et al. 2000). As topo II is able to catenate and decatenate DNA, it may be that topo II is responsible for these DNA interconnections intertwining neighbouring regions of DNA and thereby shortening and compacting chromosome arms (Fig. 1b). Indeed, a recent report suggested that topo II is required for the flexibility of the mitotic chromosome (Kawamura et al. 2010). Exogenously added topo II is able to relax the mitotic chromosome by resolving double-stranded DNA entanglements within the chromosome. Thus, topo II might play an important role in helping the chromosome to withstand forces generated by the mitotic spindle. This observation might also explain why topo II stays loosely associated with chromosomes even when chromosome condensation is completed.
In many cell types, topo II accumulates at mitotic centromeres and remains there until early anaphase (Taagepera et al. 1993; Gorbsky 1994; Christensen et al. 2002; Null et al. 2002). High doses of the topo II inhibitor ICRF 193 result in decompaction of centromeric chromatin and, as a consequence, defects in kinetochore structure (Rattner et al. 1996). In budding yeast, deSUMOylation of topo II is required for centromere cohesion. Mutation of SMT4, the isopeptidase that deconjugates SUMO, leads to precocious sister chromatid separation. This defect could be suppressed either by overexpression of yeast Top $2 p$ or by mutating all candidate SUMOylation sites on Top2p (Bachant et al. 2002). These studies clearly suggest a specific regulatory pathway that links topo II function to cohesion at centromeres. This requirement could be indirect, where topo II activity enables centromere compaction and assembly of the kinetochore. Alternatively, topo II might mediate the decatenation of sister DNA strands at the centromere. A role for catenated sister DNA strands in cohesion has been suggested (Murray and Szostak 1985) but initially discounted because yeast minichromosome segregation occurs normally during cell-cycle arrest in cells lacking topo II (Koshland and Hartwell 1987). Recently, a role for topo II in centromeric decatenation has re-emerged. In cells with defective sister chromatid cohesin (see below), treatment with a topo II inhibitor rescues cohesion (Wang et al. 2010). These authors suggest that a low amount of sister chromatid catenation persists through metaphase and is only resolved once cohesin is removed. Clearly, the different contributions of topo II to chromosome compaction and cohesion must be resolved. The effects of mutating SMT4 (Bachant et al. 2002) suggest that different functions may be defined by specific post-translational modifications and that these might provide an avenue for further study.

The condensins and condensation

Another major component of the scaffold is condensin, a complex consisting of five different subunits. The two core subunits, SMC2 and SMC4, belong to the SMC family of proteins. SMC proteins possess two nucleotide binding Walker A and B domains that are separated by two coiled-coiled motifs. Each SMC monomer folds back on itself and forms an antiparallel coiled-coil, creating an ATP-binding 'head' domain at one end and a 'hinge' 
domain at the other. The two SMC subunits interact with each other at the hinge region adopting a V-like structure (for review, see (Hirano 2006)). Condensin exists in two forms, condensins I and II, which differ in their three non-SMC subunits. Condensin I contains CAPG (chromosome-associated protein G), $\mathrm{CAPH}$ and CAPD2, which form a subcomplex and are thought to bind to the head region of the SMC subunits. Condensin II not only contains SMC2 and 4 but also contains a second set of nonSMC subunits CAPG2, CAPH2 and CAPD3.

Pioneering work in frog extracts implicated these evolutionarily conserved complexes in mitotic chromatin condensation. Antibodies against SMC2/4 block chromosome condensation in this system and also induce decondensation when added to already condensed chromosomes (Hirano and Mitchison 1994). In vitro purified condensin complexes have been shown to bind double-stranded DNA and possess ATP-dependent supercoiling activity and a DNA-stimulated ATPase activity (for review, see (Hirano 2006)). In vivo, the condensin complexes associate with chromatin during mitosis. Condensin II is nuclear throughout the cell cycle and associates with chromatin upon mitotic entry. In contrast, condensin I is cytoplasmic and only interacts with chromatin upon nuclear envelope breakdown. Condensins I and II associate with chromosomes independently of each other (Hirota et al. 2004; Ono et al. 2004) and do not associate with chromatin randomly: condensin I seems to bind to specific chromatin regions in an alternating fashion with condensin II (Ono et al. 2003, 2004). Condensin I may bind to histones $\mathrm{H} 1$ and $\mathrm{H} 3$, although how this binding mode results in localization to specific subregions of the chromosome is not known (Ball et al. 2002). Condensin II has recently been found to bind to mono-methylated histone H4 (Liu et al. 2010). Before mitosis histone $\mathrm{H} 4$ methylation is removed by the demethylase Phf8 and only when Phf8 is degraded can methylation occur and condensin II bind to chromatin. Condensin II loading also seems to require protein phosphatase 2A (PP2A) (Takemoto et al. 2009). The interaction between condensin II and PP2A does not depend on the catalytic activity of PP2A. The binding mechanism is currently unknown.

FRAP experiments revealed that the binding dynamics of the two condensin complexes are quite different. While condensin II binds stably to mitotic chromatin, condensin I exchanges dynamically from mitotic chromosomes (Gerlich et al. 2006). These differences in localization and physical properties might point to the fact that condensins I and II have distinct roles in mitotic chromosome architecture. Indeed, condensin I depletion leads to mitotic chromosomes appearing more swollen, while condensin II depletion makes chromosomes look curly (Ono et al. 2003). The structural basis for these differences is not yet clear.

Although initial studies in Xenopus and budding yeast suggested that condensins are essential for mitotic chromatin compaction, subsequent studies in other model systems challenged this view. Smc4 mutants in Drosophila show no defect in chromosome shortening (Steffensen et al. 2001). In DT40 cells, overall chromosome condensation appears to be normal in the absence of SMC2 and thus the absence of both condensins, but chromosomes are mechanically fragile and unable to withstand hypotonic swelling (Hudson et al. 2003). Chromosomes in vertebrate cells depleted of condensin by RNA interference (RNAi) also form but stretch abnormally when attached to the mitotic spindle (Gerlich et al. 2006). The assembly of chromosomes in prophase is also perturbed after condensin removal in Caenorhabditis elegans, DT40 and vertebrate cells (Hudson et al. 2003; Ono et al. 2004; Hagstrom et al. 2002; Kaitna et al. 2002). As significant chromosome compaction occurs before condensin I binding, it comes as no surprise that in condensin-I-depleted cells, chromosome compaction is normal. Thus, condensins seem to stabilise chromosome structure but do not seem to be the major requirement for chromosome assembly (Swedlow and Hirano 2003) (Fig. 1c).

The most striking phenotype associated with loss of condensin function in many organisms is aberrant anaphases. In Smc4 mutants in Drosophila, sister chromatid resolution is disrupted leading to severe chromosome segregation defects (Steffensen et al. 2001). Condensins are also essential for proper anaphase segregation in human cells and C. elegans (Gerlich et al. 2006; Hagstrom et al. 2002). This anaphase failure might be due to defects in metaphase chromosome structure and/or the failure to resolve sister chromatids properly. It may also reflect a loss of topo II function as topo II localisation is altered in condensin-depleted chromosomes (Coelho et al. 
2003; Hudson et al. 2003). In addition, the activity of extracted Drosophila topo II against an exogenous substrate is decreased following condensin RNAi (Coelho et al. 2003; Hudson et al. 2003).

One modifier of condensin function is the Kif4a (kinesin family member 4a), a 'chromokinesin' that binds to chromosome arms. Depletion of Kif4a by RNAi causes condensins I and II to partially delocalise from the chromosome axis. Similar to loss of condensin, loss of Kif4a results in an increase in anaphase bridges. However, whereas depletion of condensins I and II have minimal effect on the shape of mitotic chromosomes (Hirota et al. 2004), Kif4a depletion results in chromosome hypercondensation (Mazumdar et al. 2004). Although Kif4a physically interacts with both condensin complexes, its localisation on mitotic chromosomes only partially overlaps with condensins. Thus, the function of Kif4a is not simply to mediate the binding of condensins to chromatin, but it might act to define the spacing of condensin localization on DNA.

A number of experiments suggest that chromosome condensation is regulated by cyclin-dependent kinase 1 (CDK1) phosphorylation. Prolonged CDK1 activity (e.g. by nocodazole treatment) leads to hypercondensation of mitotic chromatin (Fig. 1a). Overexpressed or non-degradable forms of cyclin B1 and cyclin B3 can block chromosome decondensation at the end of mitosis (Parry and O'Farrell 2001). In vitro, the supercoiling activity of condensins is enhanced by CDK1 (Kimura et al. 1998) suggesting that the role of CDK1 in chromosome condensation is mediated through condensins. However, when GFPcyclin B3 was expressed in SMC2-deficient DT40 cells, chromosome compaction was maintained even after the onset of anaphase and anaphase defects normally observed in cells lacking condensin were efficiently rescued. The same phenotype was observed if protein phosphatase 1, which is thought to reverse mitotic phosphorylation, is prevented from localizing to anaphase chromosomes (Vagnarelli et al. 2006). These observations suggest a condensin independent role for CDK1 activity in chromosome condensation and prompted Vagnarelli et al. to postulate the existence of a 'regulator of chromosome architecture' (RCA) that is positively regulated by CDK1 activity as a key factor for chromosome condensation. Various screens have failed to uncover this mysterious protein (Kittler et al. 2007; Neumann et al. 2010), possibly because various parallel pathways contribute to chromosome condensation and disruption of only one pathway may not reveal a detectable phenotype.

Cohesin

After DNA replication, sister chromatids are held together by a large multisubunit complex known as cohesin. The cohesin complex consists of two SMC proteins, SMC1 and SMC3, and two non-SMC subunits-sister chromatid cohesion protein 1 (SCC1) and SCC3. Cells of higher eukaryotes contain two orthologues of SCC3, called stromal antigen (SA) 1 and SA2. Cohesin complexes in these cells contain either SA1 or SA2, but not both. All components of the cohesin complex are highly conserved and necessary for the proper function of cohesin. Genetic disruption or immunodepletion of any of the subunits causes loss of sister cohesion before anaphase and leads to frequent errors in chromosome segregation (Guacci et al. 1997; Michaelis et al. 1997; Losada et al. 1998).

In all cells, anaphase onset initiates when SCC1 is proteolytically cleaved, causing the release of cohesin and allowing the forces generated by kinetochoremicrotubule attachments to segregate chromosome pairs to daughter cells (Oliveira and Nasmyth 2010). However, in animal cells, the bulk of cohesin dissociates from chromosomes in prophase in a process that does not require the cleavage of SCC1 (Waizenegger et al. 2000; Nasmyth and Haering 2009) (Fig. 2). This 'prophase pathway' requires the activities of the Polo like kinase 1 (Plk1) and Aurora B kinases (Losada et al. 2002; Sumara et al. 2002). Plk1 can phosphorylate SCC1 and SCC3 in vitro. While the phosphorylation of SCC1 does make it more susceptible to separase cleavage, it does not lead to separase-independent dissociation of cohesin from chromatin. In contrast, phosphorylation of SCC3/SA2 is essential for removal of cohesin from chromosomes in prophase (Hauf et al. 2005). Expression of a non-phosphorylatable SA2 variant only abolishes prophase dissociation of cohesin, leaving cohesin removal by separase activity at anaphase onset intact (Hauf et al. 2005). Removal of cohesin also requires wings apart-like (Wapl) and precocious dissociation of sisters 5 (Pds5), two proteins that physically interact with the cohesin complex and play a key role in promoting the prophase resolution 

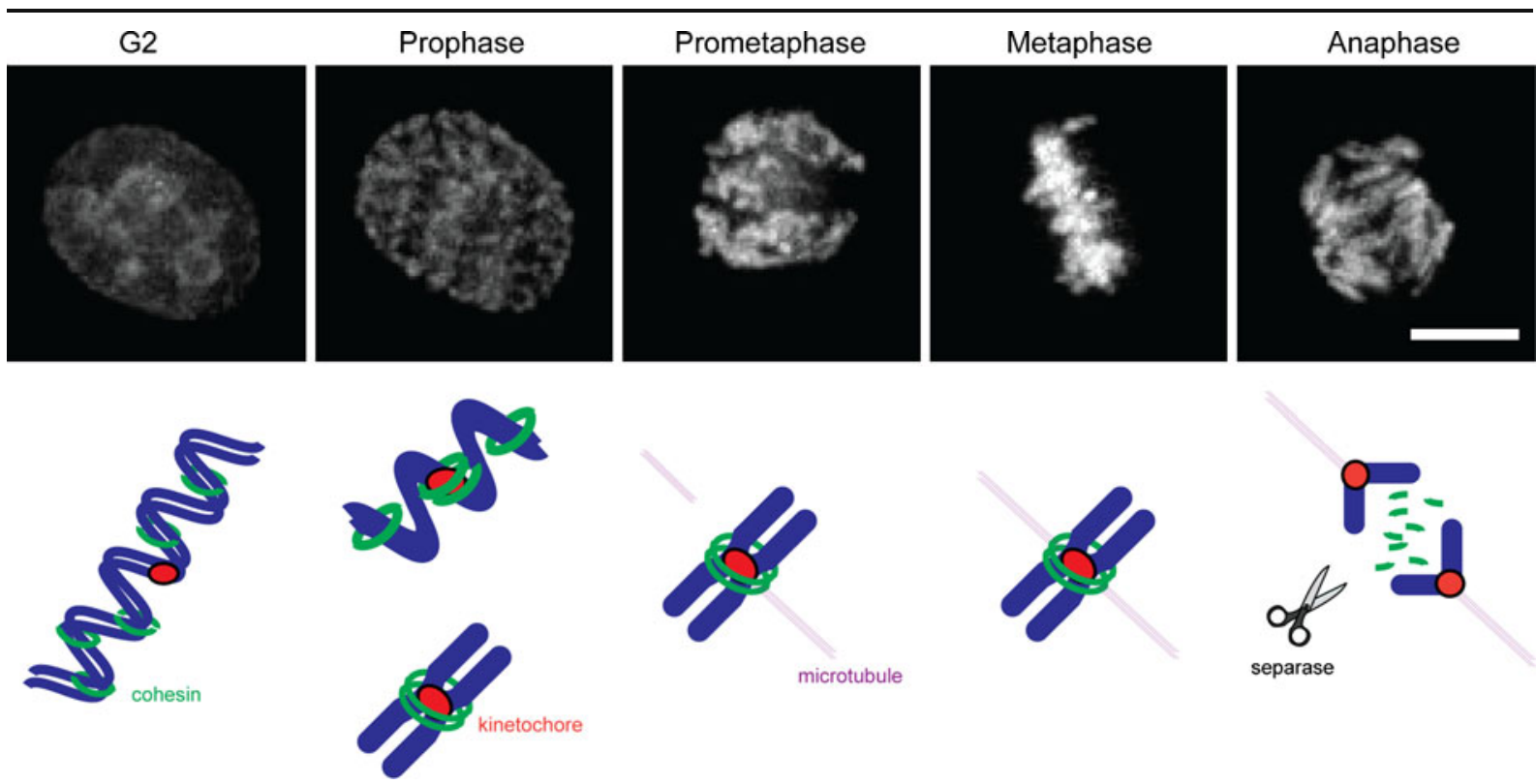

Fig. 2 Changes in cohesin association with chromatin during mitosis. During G2 sister chromatids are held together by cohesin. Upon entry into mitosis, most of the arm cohesin is removed, sister chromatids, resolve and condense. After attachment of kineto-

process (Gandhi et al. 2006; Kueng et al. 2006; Shintomi and Hirano 2009).

After cohesin is removed from chromosome arms during prophase, it persists at the centromere. Shugoshin 1 (Sgo1) is thought to protect a centromeric population of cohesin from its phosphorylation by Plk1, thereby allowing cohesin to persist at centromeres until the onset of anaphase (Kitajima et al. 2006). In Wapl- or Pds5-depleted extracts, Sgo1 does not only localize to the centromere but also shows discrete axial distribution along the entire length of the chromosome (Shintomi and Hirano 2009). However, absence of Sgol alone is not sufficient for cohesin to dissociate from chromosome arms. Wapl and Pds5 are absolutely essential for the loss of arm cohesin. Persistence of cohesin on chromosome arms caused by depletion of Plk1 can, however, be alleviated by co-depletion of Sgol (Shintomi and Hirano 2009). Plk1 and Wapl may, therefore, promote dissociation of cohesin from chromosome arms by distinct mechanisms.

Removal of cohesin is also dependent on condensin as RNAi against condensin I subunit CAPD2 results in persistence of chromosome arm cohesin (Hirota et al. 2004). How condensin participates in the removal of arm cohesin is unclear. A recent report suggests a role chores to microtubules emanating from opposite spindle poles, centromeric cohesin is cleaved by separase and sister chromatids are segregated in anaphase. Images in the upper panels show chromosomal DNA marked with H2B-GFP. Scale bar $5 \mu \mathrm{m}$

for condensin in removal of cohesin that remains after separase cleavage and anaphase onset (Renshaw et al. 2010). The authors propose that the recoiling of stretched chromosomes during anaphase is mediated by condensin and generates forces that remove residual cohesin. A similar mechanism is conceivable for cohesin removal before anaphase onset, as spindle forces are counteracted by condensin during prometaphase and metaphase (Gerlich et al. 2006). The influence of condensin on cohesin removal might also be more direct. During meiotic divisions in Saccharomyces cerevisiae, condensin promotes the ability Cdc5 (the $S$. cerevisiae Plk1 orthologue) to localize to chromosomes and to phosphorylate the meioticspecific cohesin subunit Rec8 (Yu and Koshland 2005). Similarly, condensin could aid the prophase pathway of cohesin removal in human cells.

Although cohesin removal before anaphase onset is a highly regulated and complex process, it is not clear what purpose it serves. The failure to remove cohesin in prophase and prometaphase does not lead to anaphase defects (Hauf et al. 2005) nor does it interfere with condensin binding (Losada et al. 2002). Centromere DNA decatenation depends on cohesin removal and is required for mammalian cell division (Wang et al. 2010). Cohesin removal prior to anaphase might 
facilitate sister chromatid decatenation. Although the failure to remove cohesin does not affect condensin loading, it might modulate the ability of condensin and topo II to influence condensation thereby changing the properties of mitotic chromosomes.

Despite detailed understanding of the structure and function of condensins and cohesins as single molecules, we still have little data on how they physically and functionally interact on chromatin. Further analysis in this context is certainly a priority for future work.

\section{Histone modifications}

Most studies addressing mitotic chromosome structure have focused on the non-histone protein components of mitotic chromosomes. However, histones, the fundamental building blocks of chromatin, are highly modified during mitosis. Most of these modifications have been associated with transcriptional regulation or assembly of nucleosomes during replication. Nonetheless, phosphorylation of histone H3 by Aurora B, a hallmark of mitotic chromatin, has been shown to regulate the chromatin binding of heterochromatin protein 1 (HP1), a protein involved in heterochromatin formation (Hirota et al. 2005). HP1 typically dissociates from chromosome arms during mitosis but remains chromosome-bound when phosphorylation of histone $\mathrm{H} 3$ serine 10 is inhibited. During mitosis, centromeric localization of HP1 is preserved and is critical for maintaining cohesin at centromeres (Bernard et al. 2001; Nonaka et al. 2002). It is, therefore, conceivable that dissociation of HP1 from chromosome arms contributes to the release of cohesin during prophase. Interestingly, histone $\mathrm{H} 3$ phosphorylation also regulates chromatin binding of the splicing factors SRp20 and ASF/SF2 to mitotic chromosomes (Loomis et al. 2009). When Aurora B is inhibited, SRp20 and ASF/SF2 stay on mitotic chromatin together with HP1 proteins.

Histone modifications might mediate the binding of non-histone protein binding to specific regions of chromatin. Histone $\mathrm{H} 3$ serine 10 phosphorylation coincides with chromosome condensation and could be required for condensin binding to chromatin. However, the requirement of Aurora B for condensin loading is quite variable in different organisms (Giet and Glover 2001; Hagstrom et al. 2002; Kaitna et al. 2002; Lipp et al. 2007; Takemoto et al. 2007), and biochemical data suggest that histone H3 phosphor- ylation is unlikely to be the determinant of condensin binding to chromatin (de la Barre et al. 2001). As noted above, the condensin II subunit CAPD3 binds the mono-methylated tail of histone $\mathrm{H} 4$ (Liu et al. 2010). This suggests that other non-histone proteins like condensin I and Kif4a may recognise mitosisspecific histone modifications.

Post-translational modification of histones might play a more general role in mitotic chromosome compaction. Modification of histone tails will cause regional changes in the distribution of charge in chromatin, thereby causing condensation or decondensation (Fig. 1d). As noted above, higher-order chromosome structure is quite sensitive to changes in ionic concentrations. Similarly, changes in histone tail acetylation, methylation and phosphorylation may be able to modify interactions between nucleosomes and/or chromatin fibres. We note that the unidentified mitotic chromatin condensation factor RCA (Vagnarelli et al. 2006), which is sensitive to the activity of mitotic kinases, might in fact be a combination of post-translational histone modifications.

Influence of chromatin on microtubule dynamics

Once chromosomes are attached to microtubules emanating from both spindles poles, they are transported to the middle of the cell, align and form a metaphase plate about halfway between the spindle poles. Chromosomes oscillate on the metaphase plate for many minutes until anaphase initiates. These movements are accompanied by changes in the distance between sister kinetochores, commonly referred to as 'breathing'.

The oscillation and breathing of kinetochores is thought to be caused by the assembly and disassembly of kinetochore microtubules. Pulling forces generated by interactions between spindle microtubules and kinetochores are counteracted by stretching of centromeric chromatin (Skibbens et al. 1995). The distance between kinetochore pairs, often referred to as 'tension', results from the force generated by kinetochores attached to microtubule ends and any resistance provided by the centromeric chromatin. Although condensin is dispensable for the normal level of compaction of centromeres, condensin depletion increases the interkinetochore distance at the metaphase plate and thus likely affects the stiffness of centromeric chromatin (Gerlich et al. 2006; Ribeiro et al. 2009). Cohesin, and possibly DNA catenation, would also be expected to 
contribute to centromeric chromatin stiffness, as they both restrain sister centromere separation.

These results raise the question of how the stiffness of centromeric chromatin is defined, whether it changes during mitosis and whether dynamics in stiffness affect the properties of kinetochores. A recent live cell-based kinetochore tracking assay suggests a dynamic control of centromere stiffness. Time-lapse 3D images of HeLa cells stably expressing CENPA-GFP were recorded and the positions and movements of kinetochores tracked using an automated data processing pipeline (Jaqaman et al. 2010). This first study has focussed on aligned kinetochore pairs oscillating on the metaphase plate, as these should provide a defined system for perturbation analysis. Kinetochore pairs in control cells oscillate on the metaphase plate, with a well-defined speed but a broad range of periods, suggesting that the period is set by a combination of regular and stochastic oscillators. As suggested previously (Gerlich et al. 2006; Ribeiro et al. 2009), depletion of condensin increased the length of oscillation period consistent with a reduction in centromeric chromatin stiffness. Depletion of separase, the enzyme that degrades cohesin, decreased oscillation periods, suggesting an increase in chromatin stiffness, most likely because of an increase of cohesin that crosslinks centromeric chromatin. This result suggests that the levels of cohesin on centromeric chromatin are maintained at a steady state, through a competition of assembly via an unknown mechanism, and disassembly by separase activity. By contrast, the role of the microtubule depolymerases, the enzymes that generate force at kinetochores, is limited to defining the speed of kinetochores, as depletion of the motor proteins MCAK and Kif18A has no significant effect on the oscillation period. Moreover, the oscillation speed is regulated, being highest during prometaphase and decreasing throughout metaphase, reaching a minimum just before anaphase. This regulation is lost when chromatin is made too stiffthrough separase depletion-or too relaxed-through condensin depletion. These data imply that centromere stiffness sets the force generated by microtubule motors. How this occurs is unknown, but one possibility is that centromere stiffness affects the access of centromeric signalling molecules to condensin and cohesin and perhaps other components of the centromere. In the future, it will be important to determine if centromere stiffness affects the regulation of force generating molecules at kinetochores.

\section{Conclusions}

During mitosis, chromosomes have to fulfil several requirements. They have to be short and compact relative to the length of the mitotic spindle so that segregation is physically possible but still be flexible enough to withstand the forces of the mitotic spindle. A variety of research areas have contributed to our understanding how that is achieved. Biophysical experiments indicate that the basic organization of the mitotic chromosome resembles a chromatin mesh held together by a combination of DNA and non-DNA crosslinks (Marko 2008). Proteins such as topo II or condensins contribute to the physical properties of the chromosome ensuring rigidity and elasticity. Although a fixed rigid scaffold seems unlikely, a proteinaceous network clearly functions to drive compaction. A combination of catenation, protein ATPases and electrostatic interactions may all combine to form the mitotic chromosome. New assays that probe chromatin structure inside living cells will be required to assess the combined effects of these different components of the chromosome. A recent example using Förster resonance energy transfer between histones reports on differences in chromatin compaction in living cells and enables direct measurements of changes in compaction under a variety of perturbations (Lleres et al. 2009). This type of biophysical assay, combined with modern biochemical methods, should help reveal how different chromosome complexes act, are coordinated and interact.

Acknowledgements We thank Iain Porter for images of mitotic chromosome spreads and Rachael Rutkowski for critical comments on the manuscript. Work on cell division in our laboratory is funded by the BBSRC (BB/H013024 and BB/G01518X).

Open Access This article is distributed under the terms of the Creative Commons Attribution Noncommercial License which permits any noncommercial use, distribution, and reproduction in any medium, provided the original author(s) and source are credited.

\section{References}

Adachi Y, Luke M, Laemmli UK (1991) Chromosome assembly in vitro: topoisomerase II is required for condensation. Cell 64:137-148

Bachant J, Alcasabas A, Blat Y, Kleckner N, Elledge SJ (2002) The SUMO-1 isopeptidase Smt4 is linked to centromeric cohesion through SUMO-1 modification of DNA topoisomerase II. Mol Cell 9:1169-1182 
Ball AR Jr, Schmiesing JA, Zhou C et al (2002) Identification of a chromosome-targeting domain in the human condensin subunit CNAP1/hCAP-D2/Eg7. Mol Cell Biol 22:5769-5781

Belmont AS, Sedat JW, Agard DA (1987) A three-dimensional approach to mitotic chromosome structure: evidence for a complex hierarchical organization. J Cell Biol 105:77-92

Belmont AS, Braunfeld MB, Sedat JW, Agard DA (1989) Large-scale chromatin structural domains within mitotic and interphase chromosomes in vivo and in vitro. Chromosoma 98:129-143

Belmont AS, Li G, Sudlow G, Robinett C (1999) Visualization of large-scale chromatin structure and dynamics using the lac operator/lac repressor reporter system. Methods Cell Biol 58:203-222

Bernard P, Maure JF, Partridge JF et al (2001) Requirement of heterochromatin for cohesion at centromeres. Science 294:2539-2542

Christensen MO, Larsen MK, Barthelmes HU et al (2002) Dynamics of human DNA topoisomerases IIalpha and IIbeta in living cells. J Cell Biol 157:31-44

Coelho PA, Queiroz-Machado J, Sunkel CE (2003) Condensindependent localisation of topoisomerase II to an axial chromosomal structure is required for sister chromatid resolution during mitosis. J Cell Sci 116:4763-4776

de la Barre AE, Angelov D, Molla A, Dimitrov S (2001) The $\mathrm{N}$-terminus of histone $\mathrm{H} 2 \mathrm{~B}$, but not that of histone $\mathrm{H} 3$ or its phosphorylation, is essential for chromosome condensation. EMBO J 20:6383-6393

Earnshaw WC, Heck MM (1985) Localization of topoisomerase II in mitotic chromosomes. J Cell Biol 100:1716-1725

Earnshaw WC, Laemmli UK (1983) Architecture of metaphase chromosomes and chromosome scaffolds. J Cell Biol 96:84-93

Earnshaw WC, Halligan B, Cooke CA, Heck MM, Liu LF (1985) Topoisomerase II is a structural component of mitotic chromosome scaffolds. J Cell Biol 100:1706-1715

Gandhi R, Gillespie PJ, Hirano T (2006) Human Wapl is a cohesin-binding protein that promotes sister-chromatid resolution in mitotic prophase. Curr Biol 16:2406-2417

Gasser SM, Laroche T, Falquet J, Boy de la Tour E, Laemmli UK (1986) Metaphase chromosome structure. Involvement of topoisomerase II. J Mol Biol 188:613-629

Gerlich D, Hirota T, Koch B, Peters JM, Ellenberg J (2006) Condensin I stabilizes chromosomes mechanically through a dynamic interaction in live cells. Curr Biol 16:333-344

Giet R, Glover DM (2001) Drosophila aurora B kinase is required for histone $\mathrm{H} 3$ phosphorylation and condensin recruitment during chromosome condensation and to organize the central spindle during cytokinesis. J Cell Biol 152:669-682

Gorbsky GJ (1994) Cell cycle progression and chromosome segregation in mammalian cells cultured in the presence of the topoisomerase II inhibitors ICRF-187 [(+)-1,2-bis(3,5dioxopiperazinyl-1-yl)propane; ADR-529] and ICRF-159 (Razoxane). Cancer Res 54:1042-1048

Guacci V, Koshland D, Strunnikov A (1997) A direct link between sister chromatid cohesion and chromosome condensation revealed through the analysis of MCD1 in S. cerevisiae. Cell 91:47-57

Hagstrom KA, Holmes VF, Cozzarelli NR, Meyer BJ (2002) C. elegans condensin promotes mitotic chromosome archi- tecture, centromere organization, and sister chromatid segregation during mitosis and meiosis. Genes Dev 16:729-742

Hauf S, Roitinger E, Koch B et al (2005) Dissociation of cohesin from chromosome arms and loss of arm cohesion during early mitosis depends on phosphorylation of SA2. PLoS Biol 3:e69

Hirano T (2006) At the heart of the chromosome: SMC proteins in action. Nat Rev Mol Cell Biol 7:311-322

Hirano T, Mitchison TJ (1991) Cell cycle control of higherorder chromatin assembly around naked DNA in vitro. $\mathrm{J}$ Cell Biol 115:1479-1489

Hirano T, Mitchison TJ (1993) Topoisomerase II does not play a scaffolding role in the organization of mitotic chromosomes assembled in Xenopus egg extracts. J Cell Biol 120:601-612

Hirano T, Mitchison TJ (1994) A heterodimeric coiled-coil protein required for mitotic chromosome condensation in vitro. Cell 79:449-458

Hirano T, Funahashi S, Uemura T, Yanagida M (1986) Isolation and characterization of Schizosaccharomyces pombe cutmutants that block nuclear division but not cytokinesis. EMBO J 5:2973-2979

Hirota T, Gerlich D, Koch B, Ellenberg J, Peters JM (2004) Distinct functions of condensin I and II in mitotic chromosome assembly. J Cell Sci 117:6435-6445

Hirota T, Lipp JJ, Toh BH, Peters JM (2005) Histone H3 serine 10 phosphorylation by Aurora B causes HP1 dissociation from heterochromatin. Nature 438:1176-1180

Hudson DF, Vagnarelli P, Gassmann R, Earnshaw WC (2003) Condensin is required for nonhistone protein assembly and structural integrity of vertebrate mitotic chromosomes. Dev Cell 5:323-336

Jaqaman K, King EM, Amaro AC et al (2010) Kinetochore alignment within the metaphase plate is regulated by centromere stiffness and microtubule depolymerases. J Cell Biol 188:665-679

Kaitna S, Pasierbek P, Jantsch M, Loidl J, Glotzer M (2002) The aurora B kinase AIR-2 regulates kinetochores during mitosis and is required for separation of homologous chromosomes during meiosis. Curr Biol 12:798-812

Kawamura R, Pope LH, Christensen MO et al (2010) Mitotic chromosomes are constrained by topoisomerase II-sensitive DNA entanglements. J Cell Biol 188:653-663

Khoudoli GA, Gillespie PJ, Stewart G et al (2008) Temporal profiling of the chromatin proteome reveals system-wide responses to replication inhibition. Curr Biol 18:838-843

Kimura K, Hirano M, Kobayashi R, Hirano T (1998) Phosphorylation and activation of $13 \mathrm{~S}$ condensin by $\mathrm{Cdc} 2$ in vitro. Science 282:487-490

Kitajima TS, Sakuno T, Ishiguro K et al (2006) Shugoshin collaborates with protein phosphatase $2 \mathrm{~A}$ to protect cohesin. Nature 441:46-52

Kittler R, Pelletier L, Heninger AK et al (2007) Genome-scale RNAi profiling of cell division in human tissue culture cells. Nat Cell Biol 9:1401-1412

Koshland D, Hartwell LH (1987) The structure of sister minichromosome DNA before anaphase in Saccharomyces cerevisiae. Science 238:1713-1716

Kueng S, Hegemann B, Peters BH et al (2006) Wapl controls the dynamic association of cohesin with chromatin. Cell 127:955-967 
Laemmli UK, Cheng SM, Adolph KW et al (1978) Metaphase chromosome structure: the role of nonhistone proteins. Cold Spring Harb Symp Quant Biol 42(Pt 1):351-360

Lewis CD, Laemmli UK (1982) Higher order metaphase chromosome structure: evidence for metalloprotein interactions. Cell 29:171-181

Lipp JJ, Hirota T, Poser I, Peters JM (2007) Aurora B controls the association of condensin I but not condensin II with mitotic chromosomes. J Cell Sci 120:1245-1255

Liu W, Tanasa B, Tyurina OV et al (2010) PHF8 mediates histone $\mathrm{H} 4$ lysine 20 demethylation events involved in cell cycle progression. Nature 466:508-512

Lleres D, James J, Swift S, Norman DG, Lamond AI (2009) Quantitative analysis of chromatin compaction in living cells using FLIM-FRET. J Cell Biol 187:481-496

Loomis RJ, Naoe Y, Parker JB et al (2009) Chromatin binding of SRp20 and ASF/SF2 and dissociation from mitotic chromosomes is modulated by histone $\mathrm{H} 3$ serine 10 phosphorylation. Mol Cell 33:450-461

Losada A, Hirano M, Hirano T (1998) Identification of Xenopus SMC protein complexes required for sister chromatid cohesion. Genes Dev 12:1986-1997

Losada A, Hirano M, Hirano T (2002) Cohesin release is required for sister chromatid resolution, but not for condensin-mediated compaction, at the onset of mitosis. Genes Dev 16:3004-3016

Marko JF (2008) Micromechanical studies of mitotic chromosomes. Chromosome Res 16:469-497

Marsden MP, Laemmli UK (1979) Metaphase chromosome structure: evidence for a radial loop model. Cell 17:849858

Mazumdar M, Sundareshan S, Misteli T (2004) Human chromokinesin KIF4A functions in chromosome condensation and segregation. J Cell Biol 166:613-620

Meyer KN, Kjeldsen E, Straub T et al (1997) Cell cyclecoupled relocation of types I and II topoisomerases and modulation of catalytic enzyme activities. J Cell Biol 136:775-788

Michaelis C, Ciosk R, Nasmyth K (1997) Cohesins: chromosomal proteins that prevent premature separation of sister chromatids. Cell 91:35-45

Murnion ME, Adams RR, Callister DM et al (2001) Chromatinassociated protein phosphatase 1 regulates aurora-B and histone H3 phosphorylation. J Biol Chem 276:2665626665

Murray AW, Szostak JW (1985) Chromosome segregation in mitosis and meiosis. Annu Rev Cell Biol 1:289-315

Nasmyth K, Haering CH (2009) Cohesin: its roles and mechanisms. Annu Rev Genet 43:525-558

Neumann B, Walter T, Heriche JK et al (2010) Phenotypic profiling of the human genome by time-lapse microscopy reveals cell division genes. Nature 464:721-727

Nonaka N, Kitajima T, Yokobayashi S et al (2002) Recruitment of cohesin to heterochromatic regions by Swi6/HP1 in fission yeast. Nat Cell Biol 4:89-93

Null AP, Hudson J, Gorbsky GJ (2002) Both alpha and beta isoforms of mammalian DNA topoisomerase II associate with chromosomes in mitosis. Cell Growth Differ 13:325333

Ohta S, Bukowski-Wills JC, Sanchez-Pulido L et al (2010) The protein composition of mitotic chromosomes determined using multiclassifier combinatorial proteomics. Cell 142:810-821

Oliveira RA, Nasmyth K (2010) Getting through anaphase: splitting the sisters and beyond. Biochem Soc Trans 38:1639-1644

Ono T, Losada A, Hirano M et al (2003) Differential contributions of condensin I and condensin II to mitotic chromosome architecture in vertebrate cells. Cell 115:109-121

Ono T, Fang Y, Spector DL, Hirano T (2004) Spatial and temporal regulation of Condensins I and II in mitotic chromosome assembly in human cells. Mol Biol Cell 15:3296-3308

Parry DH, O'Farrell PH (2001) The schedule of destruction of three mitotic cyclins can dictate the timing of events during exit from mitosis. Curr Biol 11:671-683

Paulson JR, Laemmli UK (1977) The structure of histonedepleted metaphase chromosomes. Cell 12:817-828

Poirier MG, Marko JF (2002) Mitotic chromosomes are chromatin networks without a mechanically contiguous protein scaffold. Proc Natl Acad Sci USA 99:15393-15397

Poirier M, Eroglu S, Chatenay D, Marko JF (2000) Reversible and irreversible unfolding of mitotic newt chromosomes by applied force. Mol Biol Cell 11:269-276

Porter IM, McClelland SE, Khoudoli GA et al (2007) Bod1, a novel kinetochore protein required for chromosome biorientation. J Cell Biol 179:187-197

Rattner JB, Hendzel MJ, Furbee CS, Muller MT, Bazett-Jones DP (1996) Topoisomerase II alpha is associated with the mammalian centromere in a cell cycle- and speciesspecific manner and is required for proper centromere/ kinetochore structure. J Cell Biol 134:1097-1107

Renshaw MJ, Ward JJ, Kanemaki M et al (2010) Condensins promote chromosome recoiling during early anaphase to complete sister chromatid separation. Dev Cell 19:232-244

Ribeiro SA, Gatlin JC, Dong Y et al (2009) Condensin regulates the stiffness of vertebrate centromeres. Mol Biol Cell 20:2371-2380

Rieder CL, Palazzo RE (1992) Colcemid and the mitotic cycle. J Cell Sci 102(Pt 3):387-392

Saitoh N, Goldberg IG, Wood ER, Earnshaw WC (1994) ScII: an abundant chromosome scaffold protein is a member of a family of putative ATPases with an unusual predicted tertiary structure. J Cell Biol 127:303-318

Saka Y, Sutani T, Yamashita Y et al (1994) Fission yeast cut3 and cut14, members of a ubiquitous protein family, are required for chromosome condensation and segregation in mitosis. EMBO J 13:4938-4952

Sedat J, Manuelidis L (1978) A direct approach to the structure of eukaryotic chromosomes. Cold Spring Harb Symp Quant Biol 42(Pt 1):331-350

Shintomi K, Hirano T (2009) Releasing cohesin from chromosome arms in early mitosis: opposing actions of Wapl-Pds5 and Sgo1. Genes Dev 23:2224-2236

Skibbens RV, Rieder CL, Salmon ED (1995) Kinetochore motility after severing between sister centromeres using laser microsurgery: evidence that kinetochore directional instability and position is regulated by tension. J Cell Sci 108(Pt 7):2537-2548

Steffensen S, Coelho PA, Cobbe N et al (2001) A role for Drosophila SMC4 in the resolution of sister chromatids in mitosis. Curr Biol 11:295-307 
Strukov YG, Belmont AS (2009) Mitotic chromosome structure: reproducibility of folding and symmetry between sister chromatids. Biophys J 96:1617-1628

Strunnikov AV, Hogan E, Koshland D (1995) SMC2, a Saccharomyces cerevisiae gene essential for chromosome segregation and condensation, defines a subgroup within the SMC family. Genes Dev 9:587-599

Stubblefield E, Wray W (1971) Architecture of the Chinese hamster metaphase chromosome. Chromosoma 32:262-294

Sumara I, Vorlaufer E, Stukenberg PT et al (2002) The dissociation of cohesin from chromosomes in prophase is regulated by Polo-like kinase. Mol Cell 9:515-525

Swedlow JR, Hirano T (2003) The making of the mitotic chromosome: modern insights into classical questions. Mol Cell 11:557-569

Swedlow JR, Sedat JW, Agard DA (1993) Multiple chromosomal populations of topoisomerase II detected in vivo by time-lapse, three-dimensional wide-field microscopy. Cell 73:97-108

Taagepera S, Rao PN, Drake FH, Gorbsky GJ (1993) DNA topoisomerase II alpha is the major chromosome protein recognized by the mitotic phosphoprotein antibody MPM-2. Proc Natl Acad Sci USA 90:8407-8411

Takemoto A, Murayama A, Katano M et al (2007) Analysis of the role of Aurora B on the chromosomal targeting of condensin I. Nucleic Acids Res 35:2403-2412

Takemoto A, Maeshima K, Ikehara T et al (2009) The chromosomal association of condensin II is regulated by a noncatalytic function of PP2A. Nat Struct Mol Biol 16:1302-1308
Tavormina PA, Come MG, Hudson JR et al (2002) Rapid exchange of mammalian topoisomerase II alpha at kinetochores and chromosome arms in mitosis. J Cell Biol 158:23-29

Uemura T, Yanagida M (1984) Isolation of type I and II DNA topoisomerase mutants from fission yeast: single and double mutants show different phenotypes in cell growth and chromatin organization. EMBO J 3:1737-1744

Uemura T, Ohkura H, Adachi Y et al (1987) DNA topoisomerase II is required for condensation and separation of mitotic chromosomes in S. pombe. Cell 50:917-925

Vagnarelli P, Hudson DF, Ribeiro SA et al (2006) Condensin and Repo-Man-PP1 co-operate in the regulation of chromosome architecture during mitosis. Nat Cell Biol 8:1133-1142

Waizenegger IC, Hauf S, Meinke A, Peters JM (2000) Two distinct pathways remove mammalian cohesin from chromosome arms in prophase and from centromeres in anaphase. Cell 103:399-410

Wang LH, Mayer B, Stemmann O, Nigg EA (2010) Centromere DNA decatenation depends on cohesin removal and is required for mammalian cell division. J Cell Sci 123:806813

Wood ER, Earnshaw WC (1990) Mitotic chromatin condensation in vitro using somatic cell extracts and nuclei with variable levels of endogenous topoisomerase II. J Cell Biol 111:28392850

Yu HG, Koshland D (2005) Chromosome morphogenesis: condensin-dependent cohesin removal during meiosis. Cell 123:397-407 